\title{
TROMBOSE RECORRENTE DE ARTÉRIA HEPÁTICA ASSOCIADA À POSITIVIDADE DE ANTICORPO ANTICARDIOLIPINA NO PÓS-OPERATÓRIO DE TRANSPLANTE DE FÍGADO: RELATO DE CASO E REVISÃO DA LITERATURA
}

\author{
Recurrent hepatic artery thrombosis associated with positivity to the anticardiolipin antibody in \\ postoperative liver transplant: Case report and literature review
}

\author{
José Antonio Possatto Ferrer, Julia Girardi Cutovoi, Anaisa Portes Ramos, Elaine Cristina de Ataíde, \\ Ilka de Fátima Santana Ferreira Boin
}

\begin{abstract}
RESUMO
Introdução: A trombose de artéria hepática é uma complicação frequente no pós-transplante hepático (varia de 2,0$20 \%$ ), ocorrendo precocemente (30 dias após transplante) em $46,7 \%$ das vezes, e necessitando novo transplante em aproximadamente metade dos casos (53,1\%). A síndrome antifosfolípide é definida como presença de trombose arterial e/ou venosa associada a anticorpos contra fosfolipídios. A incidência de SAF foi estimada em cinco novos casos/ 100000 pessoas/ ano. A ocorrência de trombose precoce de artéria hepática pós-transplante secundária à síndrome antifosfolípide é evento raro. Métodos: Relato de caso de um paciente submetido a três transplantes hepáticos por trombose de artéria associada à positividade de anticorpo anticardiolipina. Resultados: Paciente masculino, 49 anos, cirrótico por vírus $\mathrm{C}$, sem antecedentes pessoais trombóticos, incluído em lista de transplante hepático por apresentar à tomografia computadorizada, nódulo de 4,2cm no segmento VI hepático compatível com Carcinoma Hepatocelular. Submetido a transplante hepático sob a técnica de Piggy Back. Apresentou ao Ultrassom Doppler de controle, ausência de fluxo em artéria hepática, confirmado por angiotomografia. Submetido a novo transplante no $13^{\circ}$ dia pós-operatório. Nova tomografia de abdome não identificou fluxo arterial intra-hepático, e evidenciou extensa área de infarto no terceiro pós-operatório. Iniciada investigação de trombofilias, com positividade para pesquisa do anticorpo anticardiolipina. Submetido a terceiro transplante hepático, quando foi optado pela confecção de anastomose aorto-íliaco-hepática, através da interposição de enxerto de artéria ilíaca do doador. Paciente com boa evolução clínica, apresentando fluxo arterial intra-hepático aos exames de imagem de controle. Recebeu alta hospitalar no $10^{\circ}$ dia pós-operatório. Discussão e Conclusão: Mortalidade por trombose precoce de artéria hepática de 33,3\%; tempo médio de diagnóstico em torno de sete dias; principais fatores de risco (tempo operatório prolongado, baixo peso do receptor, discordância entre sorologia do doador e receptor para CMV). Encontrada correlação entre IgM anticardiolipina e eventos trombóticos $(\mathrm{p}=0.004)$. Achados sugerem que a anticardiolipina está elevada em pacientes com insuficiência hepática e pode estar associada à patogênese da trombose arterial. Não há na literatura consenso sobre o uso profilático de anticoagulantes nesses casos.
\end{abstract}

Descritores: Artéria Hepática; Transplante; Anticorpos Anticardiolipina

\section{INTRODUÇÃO}

Instituição:

Departamento de Cirurgia da Faculdade de Ciências Médicas da Universidade Estadual de Campinas (UNICAMP).

Correspondência:

José Antonio Possatto Ferrer

Rua Carlos Chagas, 420 - Cidade Universitária, CEP 13083-878 -

Campinas/SP

Tel: (15) 99786-4910

Email: zeferrer@ig.com.br

Recebido em:09/08/2013

Aceito em: 30/09/3013
A trombose de artéria hepática (TAH) é complicação bastante temida no pós-operatório de transplante hepático, sendo importante causa de perda de enxer to e mortalidade. Pode ser dividida em duas categorias: trombose precoce (ou seja, dentro de um mês após o transplante) e tardia. Dentre todos os casos de TAH, a incidência de trombose precoce e tardia é bastante semelhante $(46,7 \%$ vs $53,3 \%) .{ }^{1}$ Sua incidência é de $4,4 \%$ entre todos os pacientes transplantados. Ocorre mais frequentemente em crianças do que em adultos $(8,3 \%$ contra $2,9 \%, \mathrm{p}<0,001)$. Apresenta mortalidade no pós-operatório de transplante hepático de $33,3 \% .^{2}$ 
Dentre os principais fatores de risco, destacam-se o CMV mismatch (doador soropositivo para CMV com receptor negativo), retransplante, uso de enxertos vasculares, tempo operatório prolongado, baixo peso do receptor e variações anatômicas. ${ }^{2}$

Entre as manifestações clínicas, destacam-se febre, fadiga e elevação dos valores das transaminases e bilirrubina. Septicemia e insuficiência hepática aguda podem ocorrer nos casos graves; entretanto, a TAH precoce pode ocorrer de forma assintomática. ${ }^{3} \mathrm{~A}$ maioria dos pacientes apresentase com insuficiência hepática aguda (30\%), necessitando de retransplante em $81 \%$ dos casos. ${ }^{4}$ A Ultrassonografia Doppler (USG-D) é muito importante para a detecção de complicações vasculares não suspeitadas, particularmente a TAH, nas primeiras duas semanas após o transplante hepático. Inicialmente, sintomas, sinais e anormalidades laboratoriais estão ausentes na TAH precoce, justificando o screening com USG-D. ${ }^{5}$

As principais modalidades de tratamento compreendem a revascularização (trombólise, trombectomia), retransplante, ou tratamento expectante. A revascularização em pacientes assintomáticos, diagnosticados por USG-D, bem como o retransplante em pacientes sintomáticos, compreende a melhor estratégia para preservação da função dos enxertos e da sobrevida. ${ }^{6}$

Anticorpos antifosfolípides (APL) são um grupo de anticorpos que exibem afinidade por fosfolípides de membrana celular carregados negativamente. Sua presença pode estar associada a aumento do risco de várias formas de trombose. Sua prevalência na população geral é estimada em 1-8\%. ${ }^{7}$ Um subgrupo de pacientes com persistência desses anticorpos é mais propenso à trombose arterial ou venosa recorrentes, abortos de repetição e trombocitopenia. Essa condição é denominada Síndrome do Anticorpo Antifosfolípide (SAF). ${ }^{8}$

Essa síndrome pode ser primária ou secundária; esta última mais associada ao Lúpus Eritematoso Sistêmico (LES) e menos frequentemente a infecções, fármacos e outras doenças. Os principais marcadores sorológicos da SAF são o anticoagulante lúpico e a anticardiolipina (ACL). ${ }^{9}$

$\mathrm{O}$ presente estudo faz o relato de caso de um paciente submetido a três transplantes hepáticos por trombose de artéria associada à positividade de anticorpo anticardiolipina.

\section{RELATO DE CASO}

Paciente do sexo masculino, 49 anos, assintomático, cirrótico por vírus $C$, com MELD-11, incluído em lista de transplante hepático por apresentar à tomografia de abdome nódulo de $4,2 \mathrm{~cm}$ no segmento VI hepático, compatível com carcinoma hepatocelular. Dosagem de Alfa-Feto Proteína de 138, bilirrubina total de 1,49 , creatinina de 0,64 , INR de 1,26, configurando MELD=11. Cintilografia óssea e tomografia de tórax mostrando ausência de lesões sugestivas de metástases. Submetido a transplante hepático ortotópico total de fígado, sob a técnica de Piggyback 3 veias, com tempo de cirurgia de cinco horas. Tempo de isquemia fria de oito horas e de isquemia quente de 50 minutos. No intra-operatório recebeu apenas quatro unidades de plasma fresco congelado, apresentando boa evolução pós-operatória.

No $5^{\circ}$ dia pós-operatório, foi realizado USG-D mostrando fígado com áreas hiperecogênicas heterogêneas na periferia dos segmentos V e VI, compatíveis com áreas de infarto; veia porta com fluxo e morfologia preservados, porém, ausência de fluxo em artéria hepática. A angiotomografia de abdome confirmou o achado ultrassonográfico. Paciente incluído em lista, submetido a retransplante no $14^{\circ}$ dia pós-operatório.

No $7^{\circ}$ dia pós-operatório, nova angiotomografia de abdome mostrou fígado com extensa área de infarto e trombose de artéria hepática. Pela recorrência da trombose, foi solicitada avaliação para trombofilia, sendo pesquisados: mutação do fator $\mathrm{V}$ de Leiden, anticoagulante Lúpico, mutação no gene da protrombina e anticorpo anticardiolipina, esse último, com resultado positivo.

Submetido a novo transplante hepático no $11^{\circ}$ dia pósoperatório. Por apresentar ausência de fluxo em artéria hepática após anastomose entre hepática comum do doador e tronco celíaco do receptor, foi confeccionada anastomose aorto-ilíaco-hepática, através da interposição de enxerto de artéria ilíaca do doador (Figura 1). Paciente apresentou boa evolução clínica, recebendo alta hospitalar no $10^{\circ}$ dia pós-operatório.

Figura 1 - Anastomose arterial aorto-ilíaco-hepática, através da

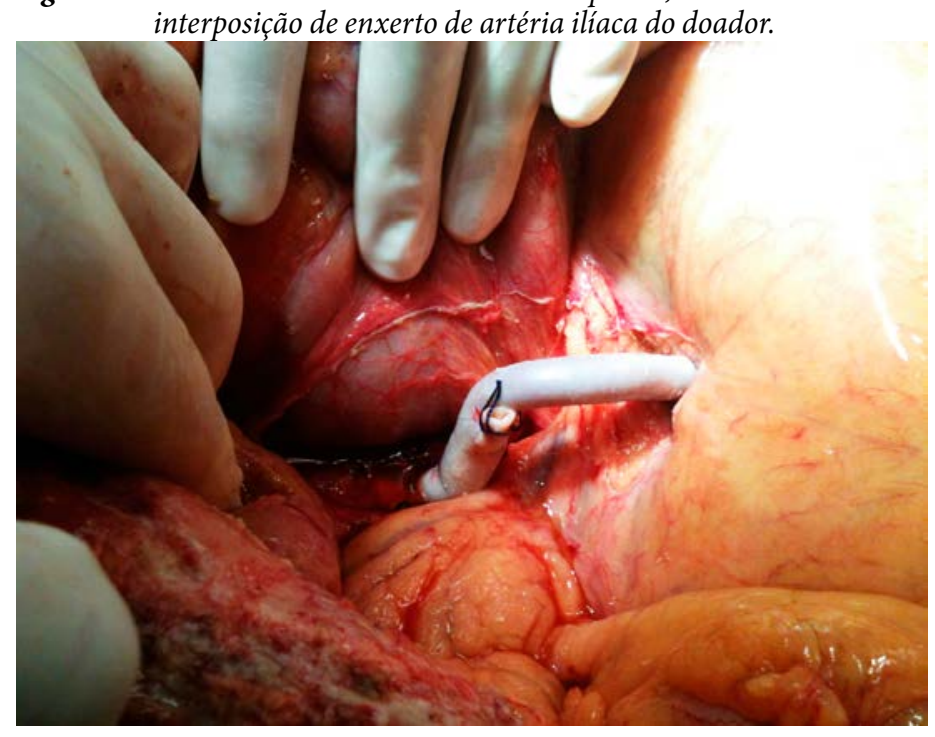

\section{DISCUSSÃO}

A presença de anticorpos antifosfolípides pode estar associada a risco trombótico. Trombose vascular do enxerto pode levar à necessidade de retransplante, aumentando a morbidade.

A presença de títulos elevados de ACL seis meses após o primeiro episódio de tromboembolismo venoso aumenta o 
risco de recorrência da trombose. Schulman et al, estudando 412 pacientes com primeiro episódio de tromboembolismo venoso recebendo anticoagulação encontrou risco de recorrência trombótica de $29 \%$ em pacientes com pesquisa positiva para ACL, comparado a $14 \%$ daqueles sem esses anticorpos $(\mathrm{p}=0,0013)$. O risco de recorrência aumentou com o título desses anticorpos. ${ }^{10}$

Em seguimento de 104 pacientes com tripla positividade para anticorpos antifosfolípides (anticoagulante lúpico, ACL e anti B2 Glicoproteina), Pengo et al encontraram incidência de 25 casos novos de eventos trombóticos $(5,3 \%$ ao ano, com incidência cumulativa após 10 anos de 37,1\%). ${ }^{11}$ A positividade com baixos títulos de ACL é achado comum em pacientes com hepatite viral crônica por vírus C (21\%), não estando associada à atividade histológica, carga viral ou resposta ao IFN- $\alpha .{ }^{8} \mathrm{Em}$ pacientes com hepatite $\mathrm{C}$, a presença de trombocitopenia, hipertensão portal e eventos trombóticos correlaciona-se com a positividade para ACL. ${ }^{12}$

Em estudo avaliando a associação entre TAH e positividade para ACL em pacientes submetidos a transplante hepático, foram estudados 28 pacientes transplantados sem TAH, sete pacientes transplantados com TAH, e 35 pacientes controle. Em 63\% dos pacientes transplantados houve positividade para ACL, estando presente em $100 \%$ daqueles com TAH, comparado a $54 \%$ daqueles sem TAH $(\mathrm{p}=0,031) .{ }^{13}$

Collier et al avaliando a presença de ACL em 132 pacientes submetidos a transplante hepático, encontraram trombose de vasos hepáticos em $16 \%$, com ACL positiva em $8,8 \%$ desses pacientes, contra $6,3 \%$ nos transplantados sem tais complicações. ${ }^{14}$

Furmanczyk-Zawiska et al estudando 33 pacientes transplantados hepáticos quanto a presença de anticorpos antifosfolípides, encontraram maior prevalência de ACL no subgrupo de alto risco trombótico (trombose de veia hepática, TAH precoce ou tardia, trombose venosa profunda associada a embolia pulmonar, trombose de veia porta). Entretanto, tal relação não foi confirmada em nova dosagem, seis meses após. Notou-se, ainda, correlação entre ACL e número de episódios trombóticos. ${ }^{7}$

Embora a existência de fenômenos trombóticos esteja relacionada nessa população, não há consenso na literatura sobre o uso de anticoagulantes de modo profilático.

\section{CONCLUSÃO}

A presença de ACL pode representar maior risco de complicações vasculares (como TAH) em pacientes transplantados hepáticos, embora, como visto acima, de maneira transitória. É necessário determinar sua importância como fator de risco para trombose e, consequentemente, perda do enxerto, principalmente em um contexto onde há desproporção entre a oferta de doadores e a demanda de receptores.

\section{ABSTRACT}

Introduction: Hepatic artery thrombosis is a frequent complication following liver transplantation (ranging from 2.0 to $20 \%$ ), occurring early in $46.7 \%$ of cases (30 days after transplant), and requiring re-transplant in about half of cases (53.1\%). Antiphospholipid syndrome (APS) is defined as the presence of arterial and/or venous thrombosis associated with antibodies directed to phospholipids. The incidence of APS was estimated in 5 new cases/100000 subjects/year. The occurrence of early hepatic artery thrombosis following transplantation secondary to antiphospholipid syndrome is a rare event. Methods: Case report of a patient undergoing three liver transplants by artery thrombosis associated with anticardiolipin antibody positivity. Results: Male patient, 49 years old, cirrhotic $\mathrm{C}$ virus, no thrombotic antecedents, included on the list for liver transplantation by presenting $4.2 \mathrm{~cm}$ nodule in liver VI segment in CT scan compatible with Hepatocellular Carcinoma. He underwent liver transplantation under Piggy back technique. In Doppler ultrasound control absence of flow in the hepatic artery, confirmed by angiotomography. Subjected to re-transplant on the 13th postoperative day. After 5 days, patient developed biliary fistula. Performed laparotomy, it was found fistulous orifice with suffering bile duct proximal to the anastomosis of the common bile duct, as well as reduced flow in the hepatic artery with ischemic liver aspect. New computerized tomography did not identify intrahepatic arterial flow, and showed extensive area of hepatic ischemia. It was initiated investigation for thrombophilia, with positive anticardiolipin antibody research. He underwent a third liver transplant, opted by aorto-iliachepatic anastomosis with interposition graft of donor iliac artery. Patient showed satisfactory clinical outcome with intra hepatic arterial flow at Doppler control. He was discharged on the 10th postoperative day. Discussion and Conclusion: $33.3 \%$ mortality due to early thrombosis of hepatic artery; 7 days of mean diagnostic time; major risk factors (prolonged operative time, low weight of the receiver, mismatch serology for CMV); findings suggest that anticardiolipin is elevated in patients with impaired hepatic function, and may be associated with the pathogenesis of arterial thrombosis although We have not found the use of this drug in patients for treatment and prevent venous thromboembolic events.

Keywords: Hepatic Artery; Transplantation; Antibodies, Anticardiolipin. 
Trombose recorrente de artéria hepática associada à positividade de anticorpo anticardiolipina no pós-operatório de transplante de fígado: relato de caso e revisão da literatura

\section{REFERÊNCIAS}

1) Stange BJ, Glanemann $M$, Nuessler NC, Settmacher $U$, Steinmüller T, Neuhaus P. Hepatic artery thrombosis after adult liver transplantation. Liver Transplantation. 2003 Jun;9(6):61220 .

2) Bekker J, Ploem S, de Jong KP. Early Hepatic Artery Thrombosis after Liver Transplantation: A Systematic Review of the Incidence, Outcome and Risk Factors. Am J Transpl. 2009;9:746-57.

3) Wu L, Zhang J, Guo Z, Tai Q, He X, Ju W et al. Hepatic Artery Thrombosis After Orthotopic Liver Transplant: A Review of the Same Institute 5 Years Later. Exp Clin Transplant. 2011 Jun;9(3):191-6.

4) Pareja E, Cortes M, Navarro R, Sanjuan F, López R, Mir J. Vascular complications after orthotopic liver transplantation: hepatic artery thrombosis. Transplant Proc. 2010 Oct;42(8):2970-2

5) Kok T, Slooff MJ, Thijn CJ, Peeters PM, Verwer R, Bijleveld CM et al. Routine Doppler ultrasound for the detection of clinically unsuspected vascular complications in the early postoperative phase after orthotopic liver transplantation. Transpl Int. 1998;11(4):272-6.

6) Sheiner et al. Selective revascularization of hepatic artery thrombosis after liver transplantation improves patient and graft survival. Transplantation. 1997 Nov 15;64(9):1295-9.

7) Furmanczyk-Zawiska A, Tronina O, Baczkowska T, Chmura A, Durlik M. The significance of antiphospholipid antibodies in liver recipients. Transplantation Proc. 2013 Jun;45(50):1983-9.
8) Leroy V, Arvieux J, Jacob MC, Maynard-Muet M, Baud M, Zarski JP. Prevalence and significance of anticardiolipin, anti-B2 glycoprotein I and anti-prothrombin antibodies in chronic hepatitis $\mathrm{C}$. $\mathrm{Br} \mathrm{J}$ Haematol. 1998;101(3):468-74.

9) Santamaria JR, Mandelli FL, Badziak D, Cavalin LC, Barros MF, Sato MS. Síndrome Antifosfolípide. An Bras Dermatol. 2005;80(3):225-39.

10) Schulman S, Svenugsson E, Grangvist S. Anticardiolipin antibodies predict early recurrence of thromboembolism and death among patients with venous thromboembolism following anticoagulant therapy. Duration of Anticoagulation Study Group. Am J Med. 1998 Apr;104(4):332-8.

11) Pengo V, Ruffatti A, Legnani C, Testa S, Fierro T, Marongiu F et al. Incidence of a first thromboembolic event in asymptomatic carriers of high-risk antiphospholipid antibody profile: a multicenter prospective study. Blood. 2011 Oct 27;118(17):4717-8.

12) ) Prieto J, Yuste JR, Beloqui O, Civeira MP, Riezu JI, Aguirre B et al. Anticardiolipin antibodies in Chronic Hepatitis C: Implication of Hepatitis C Virus as the Cause of the Antiphospholipid Syndrome. Hepatology. 1996 Feb;23(2):199-204.

13) Pascual M, Thadhani R, Laposata M, Williams WW, Farrel ML, Johnson SM et al. Anticardiolipin antibodies and hepatic artery thrombosis after liver transplantation. Transplantation. 1997 Nov 15;64(9):1361-4.

14) Collier JD, Sale J, Friend PJ, Jamieson NV, Calne RY, Alexander GJM.Graft Loss and the antiphospholipid syndrome following liver transplantation. J Hepatol. 1998;29:999-1003. 